Setting a New Standard in the Analysis of Binary Stars

K. Pavlovski, A. Tkachenko and G. Torres (eds)

EAS Publications Series, 64 (2013) 37-45

\title{
QUANTITATIVE SPECTROSCOPY OF OB STARS: FROM DWARFS TO SUPERGIANTS
}

\author{
N. Przybilla ${ }^{1}$, M.F. Nieva ${ }^{1,2}$, M. Firnstein ${ }^{2}$ and K. Butler ${ }^{3}$
}

\begin{abstract}
We discuss recent progress made in the spectral modelling of OB stars from the main sequence to evolved phases as BA-type supergiants. Non-LTE line-formation computations can now reproduce observed spectra over the entire optical and near-IR wavelength range with high confidence, facilitating stellar atmospheric parameters and elemental abundances to be determined at high accuracy and precision. An overview is given how the fundamental stellar parameters of single stars determined in our new approach compare to high-precision data derived from detached eclipsing massive binary stars. Finally, the observational constraints for a sample of Galactic objects are put in context with state-of-the-art evolution models for rotating massive stars.
\end{abstract}

\section{Introduction}

Massive OB-type stars on the main sequence and their evolved progeny, BA-type supergiants have been identified as highly valuable probes of present-day elemental abundances since long (e.g., Kilian 1992; Gies \& Lambert 1992; Venn 1995). Their enormous luminosities make BA-type supergiants accessible to quantitative spectroscopy in galaxies even well beyond the Local Group (e.g., Kudritzki et al. 2012). A full characterisation of stellar parameters and elemental abundances allows various astrophysical topics to be addressed, ranging from e.g. observational constraints on stellar evolution (Przybilla et al. 2010) over the chemical composition of the interstellar dust (Nieva \& Przybilla 2012), galactochemical evolution (Kudritzki et al. 2012) to the cosmic distance scale (Kudritzki et al. 2008).

\footnotetext{
1 Institute for Astro- and Particle Physics, University of Innsbruck, Technikerstr. 25/8, 6020 Innsbruck, Austria

2 Dr. Karl Remeis-Observatory \& ECAP, University of Erlangen-Nuremberg, Sternwartstr. 7, 96049 Bamberg, Germany

${ }^{3}$ University Observatory, University of Munich, Scheinerstr. 1, 81679 Munich, Germany
}

(C) EAS, EDP Sciences 2014 DOI: $10.1051 /$ eas/1364005 
We give here an overview of recent improvements achieved in the quantitative spectroscopy OB main-sequence stars and BA-type supergiants. Fundamental stellar parameters determined from an application of the new models and analysis methodology on single stars will be compared to high-precision data derived from detached eclipsing massive binaries (DEBs, Torres et al. 2010). In a second step the evolutionary status in particular of the BA-type supergiants - either evolving redwards from the main sequence for the first time or experiencing a blue-loop phase - will be discussed, which is currently debated (e.g., Saio et al. 2013).

\section{Observations and analysis}

The observational sample consists of bright, sharp-lined, apparently single and chemically inconspicuous stars that show no indications for the presence of a circumstellar disk. The main-sequence sample comprises a total of 29 early B-type stars discussed in detail by Nieva \& Simón-Díaz (2011, NS11) and Nieva \& Przybilla (2012, NP12). The objects are either associations members or field stars, at distances out to $\sim 400 \mathrm{pc}$ from the Sun. These are complemented by 35 BA-type supergiant stars (Firnstein \& Przybilla 2012, FP12), at distances out to $\sim 4 \mathrm{kpc}$.

High-S/N and high-resolution echelle spectra (with S/N > 250-500 typically, and resolving power $R=\lambda / \Delta \lambda \geq 40000$ ) were obtained with FEROS on the ESO/ MPG $2.2 \mathrm{~m}$ telescope at La Silla, FOCES on the $2.2 \mathrm{~m}$ telescope on Calar Alto, FIES on the $2.5 \mathrm{~m}$ Nordic Optical Telescope on La Palma, or taken from the ELODIE (Observatoire de Haute-Provence) and ESO archives (UVES/VlT). All spectra have a wide wavelength coverage $(\sim 3900-7000 \AA$, and often out to $9000 \AA)$, which is required to access all important spectroscopic indicators.

Model calculations were carried out using a hybrid non-LTE approach as discussed in detail by Przybilla et al. (2006, 2011) and Nieva \& Przybilla (2007, 2008). In brief, hydrostatic, plane-parallel and line-blanketed LTE model atmospheres were computed with ATLAs9 (Kurucz 1993). Non-LTE line formation computations were then performed on these model structures using updated versions of the Detail and Surface codes (Giddings 1981; Butler \& Giddings 1985). State-of-the-art non-LTE model atoms (see Table 1) relying on data from ab-initio computations - avoiding rough approximations wherever possible - were utilised in this step, see Przybilla (2010) for an overview of the importance of these input data. Thus, most of the astrophysically important chemical species were treated in non-LTE. Missing species were considered in LTE for completeness.

The stellar parameter and abundance determination employs an iterative analysis methodology (Przybilla et al. 2006; NP12). Numerous spectroscopic indicators such as multiple ionization equilibria and Stark-broadened profiles of the Balmer and Paschen lines are used simultaneously to derive effective temperatures $T_{\text {eff }}$ and surface gravities $\log g$. Both, high accuracy and precision can be achieved, with $1 \sigma$-uncertainties reduced to $\sim 1-2 \%$ in $T_{\text {eff }}$ (facilitating a thor-

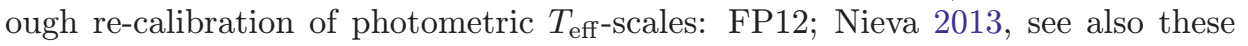
proceedings) and to $0.05-0.10 \mathrm{dex}$ in $\log g$, when the analysis is performed in a 
Table 1. Model atoms for non-LTE calculations.

\begin{tabular}{ll}
\hline \hline Ion & Model atom \\
\hline H & Przybilla \& Butler (2004) \\
He I/II & Przybilla (2005) \\
C I-IV & Przybilla et al. (2001b), Nieva \& Przybilla (2006, 2008) \\
N I/II & Przybilla \& Butler (2001), updated \\
O I/II & Przybilla et al. $(2000)$, Becker \& Butler (1988), updated \\
Ne I/II & Morel \& Butler (2008), updated \\
Mg I/II & Przybilla et al. (2001a) \\
Al II/III & Przybilla (in prep.) \\
Si III/IV & Becker \& Butler (1990), updated \\
S II/III & Vrancken et al. (1996), updated \\
Ar I/II & Butler (in prep.) \\
Ti II & Becker (1998) \\
Fe II/III & Becker (1998), Morel et al. (2006), corrected \\
\hline
\end{tabular}

self-consistent way. Absolute elemental abundances can then be constrained with unprecedented accuracy and precision: to $\sim 25 \%$ for systematic and $\sim 10-20 \%$ for random $1 \sigma$-errors. The quality of the stellar parameter determination was verified in addition by simultaneously matching the measured stellar spectral energy distributions (employing UV spectrophotometry obtained with the IUE satellite and ground-based optical and near-IR photometry) with the model fluxes. Spectroscopic distances were compared to Hipparcos distances, where feasible. A quality criterion for the elemental abundance determination was the absence of systematic trends of abundances with $T_{\text {eff }}$ or $\log g$. Finally, the model spectra were compared with the observed spectra, finding a good to excellent match throughout the entire analysed wavelength range, see Figure 1 for an example.

An application of the new models and analysis methodology to the disentangled spectra of double-lined OB-type DEBs would be highly valuable. On the one hand, this would facilitate the chemical composition of DEBs to be determined, about which little is known at present (see Pavlovski et al. 2011, and also these proceedings). On the other hand, this would allow to test our non-LTE model atoms at high detail, because of the availability of very tight and independent constraints on the atmospheric parameters of the DEB components.

\section{Fundamental parameters and a comparison to data from DEBs}

Once the basic atmospheric parameters $T_{\text {eff }}$ and $\log g$ are known, evolutionary masses $M$ of stars can be determined by comparison with the loci of stellar evolution tracks in the $T_{\text {eff }}-\log g$ plane. From these, spectroscopic distances (i.e. distance moduli) to the stars can be derived following the same strategy as in NP12, which also requires a determination of the interstellar reddening from a comparison of model and observed colours. Finally, bolometric corrections can 


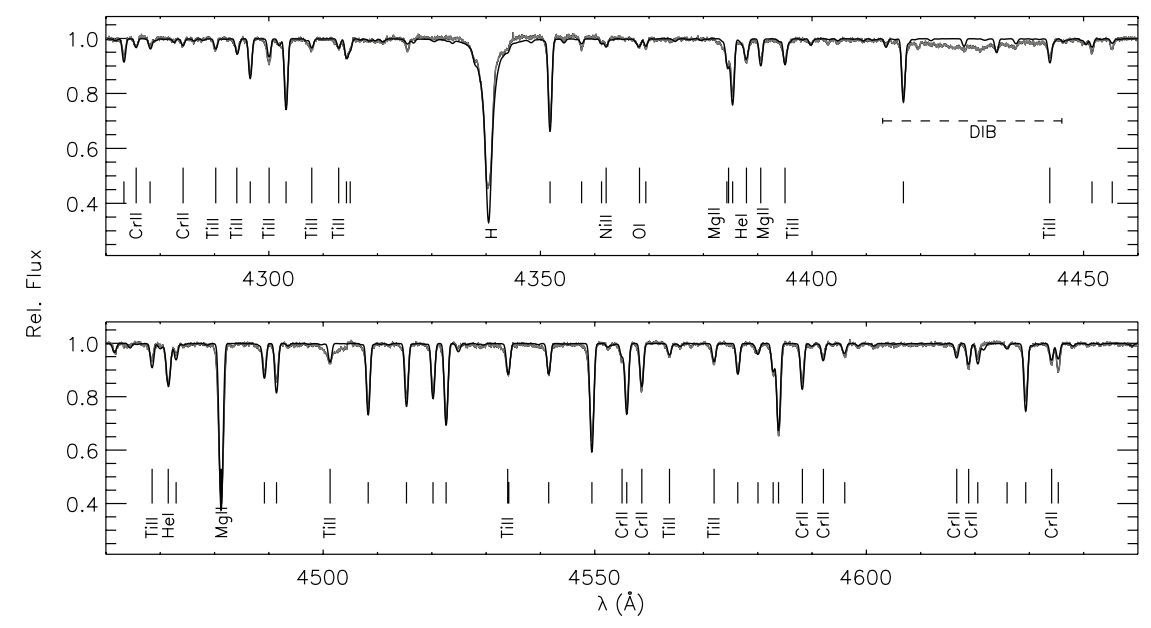

Fig. 1. Comparison of the model spectrum (black) with a high-resolution spectrum of HD 92207 (grey line). The major spectral features are identified, short vertical marks designate Fe II lines. With few exceptions, excellent agreement between theory and observation is found ( $\mathrm{H} \gamma$ is affected by the stellar wind). From Przybilla et al. (2006).

be computed from the model fluxes, providing stellar luminosities $L$ and radii $R$. Alternatively, distances of the BA-type supergiants may be derived from application of the flux-weighted gravity-luminosity relationship (Kudritzki et al. 2003, 2008).

We refer to forthcoming papers by Nieva \& Przybilla and Firnstein \& Przybilla for a discussion of the detailed results on fundamental parameters of OB stars and BA-supergiants, respectively. A preliminary summary of results on early B-stars is given in Figures 2 and 3. These show the mass-radius and mass-luminosity relationships as established from the quantitative analysis of single stars, in comparison with data derived from double-lined DEBs (Torres et al. 2010). We want to draw the attention to the extremely small error bars of the DEB data - both components of the DEBs have $M$ and $R$ determined to $\pm 3 \%$, or better. Our sample stars fit well into the trends, with error bars coming typically within a factor 2-4 to that of the DEBs. The precision in luminosity even reaches similar values as that obtained for DEBs. This opens up the possibility to improve on the number statistics and in particular to trace the regions close to the ZAMS in the $M-R$ and $M-L$ relations for the more massive objects, which so far is not covered by DEBs.

We also find a good agreement between our single star and the DEB data on early B-type dwarfs (luminosity class V) when establishing functional relationships, e.g. between spectral type and stellar mass, stellar radii or absolute visual or bolometric magnitudes, see Figure 4 for an example. Interestingly, these high-precision data differ from relations promoted in the astrophysical standard 


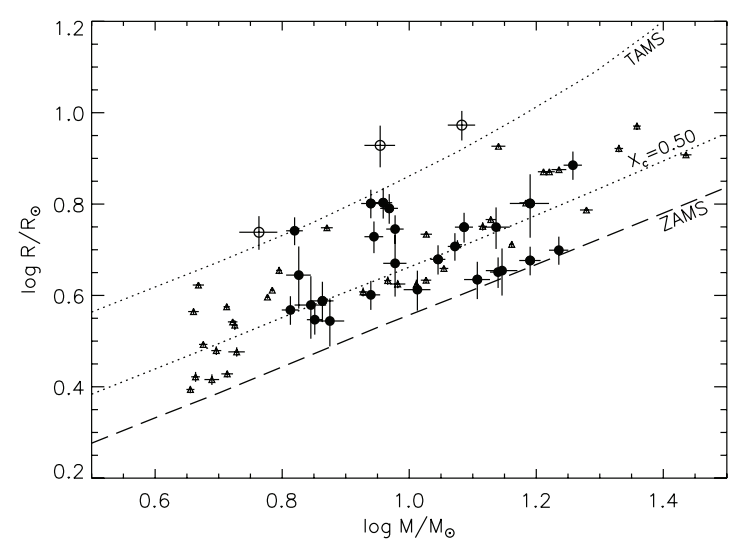

Fig. 2. Mass-radius relationship for early B-stars in the solar neighbourhood. Black dots denote bona-fide main-sequence objects and open thick circles objects near/beyond core-H exhaustion. Data from double-lined detached eclipsing binary stars (Torres et al. 2010) are shown for comparison (small triangles). In addition to the zero-age main sequence (ZAMS), two additional loci, for $50 \%$ core-H depletion and for the terminal-age main sequence (TAMS), are indicated by the thick/thin-dotted lines, as predicted by the stellar evolution models of Ekström et al. (2012). Error bars are $1 \sigma$-uncertainties.

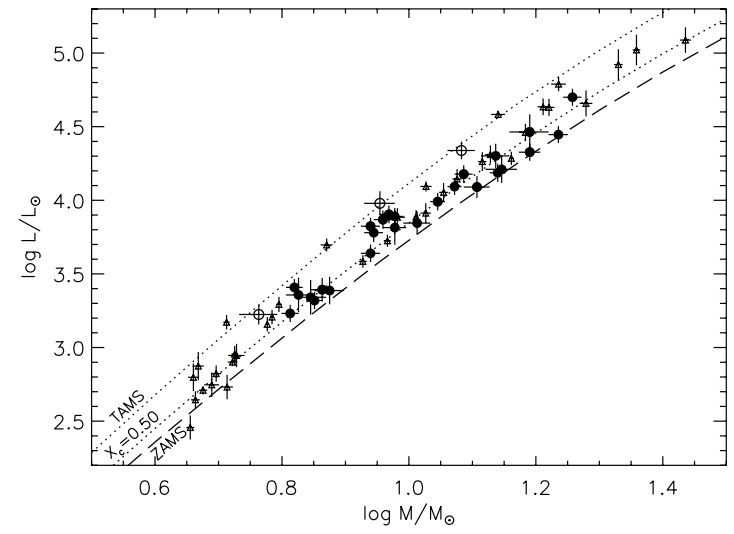

Fig. 3. Mass-luminosity relationship, see Figure 2 for details on the symbol encoding.

literature, like the Landolt-Börnstein (Schmidt-Kaler 1982) or Allen's Astrophysical Quantities (Cox 2000). The actual values are significantly smaller than the recommended reference values. Our sparse data on early B-type stars of other luminosity classes indicate that similar discrepancies occur there. We conclude, that the reference literature on fundamental stellar parameters of OB stars requires to be revised. 


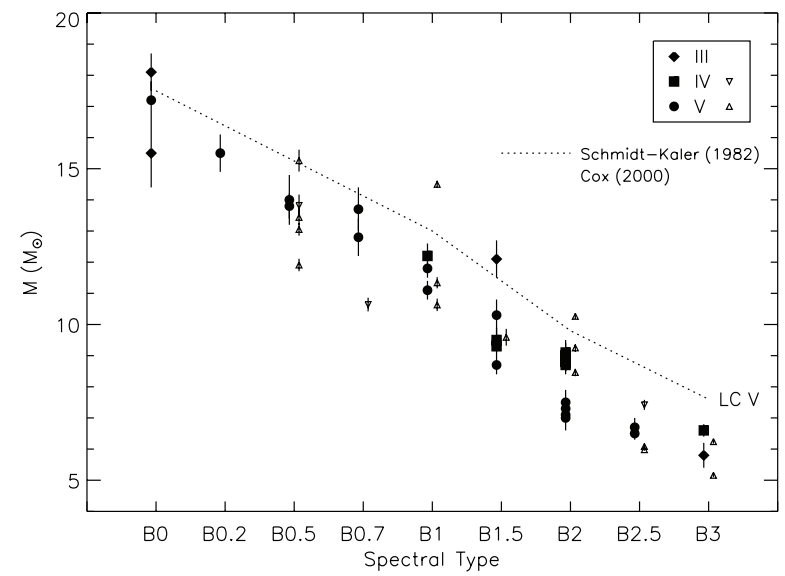

Fig. 4. Masses of early-B stars (full symbols) as a function of spectral type. Encoding of the luminosity class according to the legend, open triangles denote data derived from detached eclipsing binary stars (Torres et al. 2010). Error bars denote $1 \sigma$-uncertainties. The functional relationship for dwarfs (luminosity class V) as advocated in the astrophysical reference literature is indicated. From Nieva \& Przybilla (in prep.).

\section{Constraints on evolutionary status}

While the evolutionary status of main-sequence stars can be determined without ambiguity, that of BA-type supergiants is not clarified at present. Are they the direct progeny of main-sequence OB stars (i.e. are they crossing the HertzsprungRussell-diagram for the first time towards the red), or have they gone through the red supergiant stage before (i.e. are they on a blue loop)? A fresh view on this topic may be obtained from an accurate and precise determination of stellar parameters and elemental abundances, in particular for carbon, nitrogen and oxygen.

An indication for the absence of systematic error from stellar analyses (i.e. a quality criterion for their accuracy) may indeed be deduced objectively. It turns out that the locus of $\mathrm{N} / \mathrm{C}$ vs. $\mathrm{N} / \mathrm{O}$ abundance ratios depends only on nuclear physics (the CNO cycles), and is essentially independent of the initial stellar masses, rotation velocities and nature of the mixing processes (Przybilla et al. 2010). Indeed, only our recent investigations recover the predicted nuclear path tightly, see Figure 5. However, additional information is required to deduce indications for the evolutionary status of BA-type supergiants.

One possibility is to put surface N/C-ratios in the context of the surface gravity. This is depicted in Figure 6. From left to right, the theoretical evolution proceeds from the main sequence via the BA-type supergiant regime to the red supergiant stage, potentially entering a blue loop (where log $g$ develops towards higher values again), as for the $9 M_{\odot}$ models. For the majority of the supergiants it seems that they have evolved directly from the main sequence when compared to the stellar evolution models of Ekström et al. (2012). Their level of enrichment 


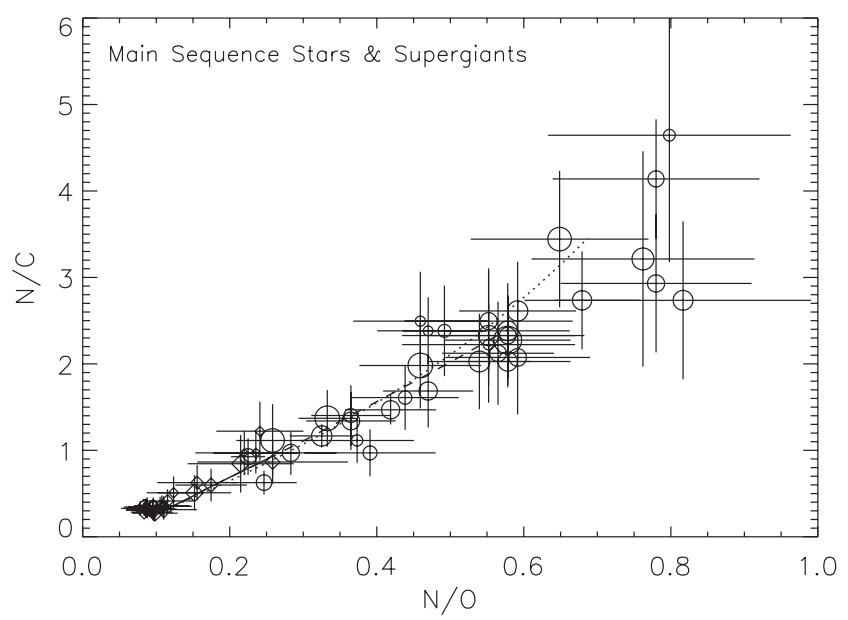

Fig. 5. N/C vs. N/O abundance ratios (by mass) in early-B dwarfs/giants (NS11; NP12; diamonds) and BA-supergiants (Przybilla et al. 2010, FP12, circles). The predicted tight trend from analytical considerations and various rotating Geneva evolution models (lines) is recovered for the first time. The symbol size is proportional to stellar mass.

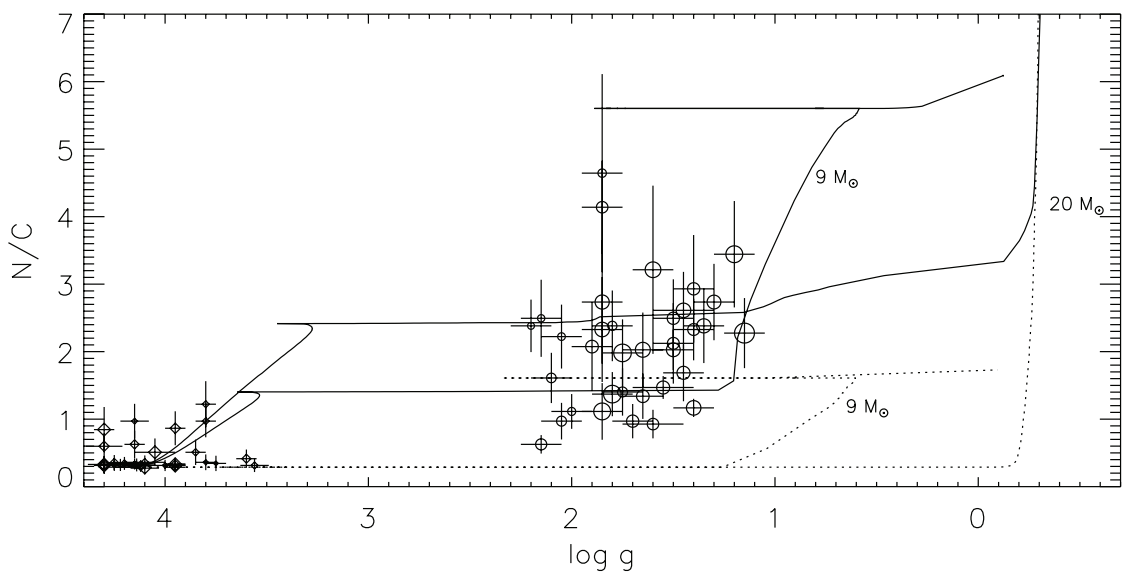

Fig. 6. Evolution of surface N/C abundances as a function of $\log g$. The same objects as in Figure 5 are shown. Geneva evolution tracks (Ekström et al. 2012) for stars of 9 and $20 M_{\odot}$ are displayed, bracketing the investigated mass range. Two cases are considered, stars initially rotating at $40 \%$ breakup velocity (full), and non-rotators (dotted lines).

with CNO-processed matter is compatible with the predictions from rotational mixing in stars with initial rotation velocities of smaller or about to $40 \%$ of the breakup velocity, implying a first redward evolution after the main-sequence stage. This, on the other hand, is contradicted by the pulsational properties of BA-type 
supergiants (Saio et al. 2013), where the observed pulsations are expected to occur only in a post-red supergiant stage. We conclude that no definite statement on the evolutionary status of the majority of BA-type supergiants can be drawn at present.

MFN acknowledges a FFL stipend from the University of Erlangen-Nuremberg and MF financial support by the Deutsche Forschungsgemeinschaft, DFG project number PR $685 / 3-1$.

\section{References}

Becker, S.R., 1998, ASP Conf. Ser., 131, 137

Becker, S.R., \& Butler, K., 1988, A\&A, 201, 232

Becker, S.R., \& Butler, K., 1990, A\&A, 235, 326

Butler, K., \& Giddings, J., 1985, Newsletter on the analysis of astronomical spectra No. 9 (Univ. London)

Cox, A.N., 2000, Allen's Astrophysical Quantities, $4^{\text {th }}$ ed. (Springer-Verlag, New York)

Ekström, S., Georgy, C., Eggenberger, P., et al., 2012, A\&A, 537, A146

Firnstein, M., \& Przybilla, N., 2012, A\&A, 543, A80 (FP12)

Giddings, J.R., 1981, Ph.D. Thesis (University College London)

Gies, D.R., \& Lambert, D.L., 1992, ApJ, 387, 673

Kilian, J., 1992, A\&A, 262, 17

Kudritzki, R.P., Bresolin, F., \& Przybilla, N., 2003, ApJ, 582, L83

Kudritzki, R.P., Urbaneja, M.A., Bresolin, F., et al., 2008, ApJ, 681, 269

Kudritzki, R.P., Urbaneja, M.A., Gazak, Z., et al., 2012, ApJ, 747, 15

Kurucz, R.L., 1993, Kurucz CD-ROM No. 13 (SAO: Cambridge, Mass.)

Morel, T., \& Butler, K., 2008, A\&A, 487, 307

Morel, T., Butler, K., Aerts, C., Neiner, C., \& Briquet, M., 2006, A\&A, 457, 651

Nieva, M.F., 2013, A\&A, 550, A26

Nieva, M.F., \& Przybilla, N., 2006, ApJ, 639, L39

Nieva, M.F., \& Przybilla, N., 2007, A\&A, 467, 295

Nieva, M.F., \& Przybilla, N., 2008, A\&A, 481, 199

Nieva, M.F., \& Przybilla, N., 2012, A\&A, 539, A143 (NP12)

Nieva, M.F., \& Simón-Díaz, S., 2011, A\&A, 532, A2 (NS11)

Pavlovski, K., Southworth, J., Tamajo, E., \& Kolbas, V., 2011, Bull. Soc. Royale Sci. Liège, 80, 714

Przybilla, N., 2005, A\&A, 443, 293

Przybilla, N., 2010, EAS Publications Series, 43, 115

Przybilla, N., \& Butler, K., 2001, A\&A, 379, 955

Przybilla, N., \& Butler, K., 2004, ApJ, 609, 1181

Przybilla, N., Butler, K., Becker, S.R., Kudritzki, R.P., \& Venn, K.A., 2000, A\&A, 359, 1085

Przybilla, N., Butler, K., Becker, S.R., \& Kudritzki, R.P., 2001a, A\&A, 369, 1009 
Przybilla, N., Butler, K., Becker, S.R., \& Kudritzki, R.P., 2006, A\&A, 445, 1099

Przybilla, N., Butler, K., \& Kudritzki, R.P., 2001b, A\&A, 379, 936

Przybilla, N., Firnstein, M., Nieva, M.F., Meynet, G., \& Maeder, A., 2010, A\&A, 517, A38

Przybilla, N., Nieva, M.F., \& Butler, K., 2008, ApJ, 688, L103

Przybilla, N., Nieva, M.F., \& Butler, K., 2011, J. Phys.: Conf. Ser., 328, 012015

Saio, H., Georgy, C., \& Meynet, G., 2013, MNRAS, 433, 1246

Schmidt-Kaler, T., 1982, in: Landolt-Börnstein, Vol. 2, Subvol. b (Springer-Verlag, Berlin)

Torres, G., Andersen, J., \& Giménez, A., 2010, A\&ARv, 18, 67

Venn, K.A., 1995, ApJS, 99, 659

Vrancken, M., Butler, K., \& Becker, S.R., 1996, A\&A, 311, 66 